\title{
IMECE2010-37369
}

\section{COMBINING VARIATION SIMULATION WITH WELDING SIMULATION FOR PREDICTION OF DEFORMATION}

\author{
Andreas Pahkamaa \\ Division of Computer Aided Design \\ Luleå University of Technology \\ SE-971 87 Luleå \\ Sweden
}

\author{
Kristina Wärmefjord \\ Department of Product and Production Development \\ Chalmers University of Technology \\ SE-412 96 Göteborg \\ Sweden
}

\author{
Lennart Karlsson \\ Division of \\ Computer Aided Design \\ Luleå University of Technology \\ SE-971 87 Luleå \\ Sweden
}

\author{
Rikard Söderberg \\ Department of Product and Production \\ Development \\ Chalmers University of Technology \\ SE-412 96 Göteborg \\ Sweden
}

\author{
John Goldak \\ Department of Mechanical \& \\ Aerospace Engineering Carleton \\ University, Ottawa \\ Canada
}

\begin{abstract}
In most variation simulations, i.e. simulations of geometric variations in assemblies, the influence from the heating and cooling processes, generated when two parts are welded together, is not taken into consideration. In most welding simulations the influence from geometric tolerances on parts is not taken into consideration, i.e. the simulations are based on nominal parts. In this paper these two aspects, both crucial for predicting the final outcome of an assembly, are combined by linking two commercial software packages for variation simulation and for welding simulation together. Monte Carlo simulation is used to generate a number of different non-nominal parts in the variation simulation software. The translation and rotation matrices, representing the deviations from the nominal geometry due to positioning error, are exported to the welding simulation software, where the effects from welding are applied. Thereafter, the results from the welding simulation are exported back to the variation simulation software in order to compute and illustrate the deviations and variations of the final subassembly. The method is applied on a simple case, a Tweld joint, with available measurements of residual stresses and deformations. The effect of the different sources of deviation on the final outcome is analyzed and the difference between welding simulations applied to nominal parts and to disturbed parts is investigated.
\end{abstract}

Keywords: variation simulations, welding simulations, tolerances, Monte Carlo, simulation driven design.

\section{INTRODUCTION}

In this very first section, a short background is given and the need of the suggested method is explained. Further, both variation simulations and welding simulations, which will be combined in this work, are described.

\subsection{Background}

In manufacturing industry, different kinds of simulation tools are used, aiming to predict the outcome of the final product. Those predictions are usually done in early stages of the product development cycle in order to compare different product concepts and process alternatives. Increased demands on sustainability drives the need for an increased use of virtual methods for verification of product and production systems.

Among the simulation tools, variation simulation is used to predict the geometrical variation in critical dimensions of a subassembly or final product. Such a simulation is based on CAD-models, or meshes in the case of non-rigid simulations. Tolerances are added to important features in the simulation model and the resulting variation in the final subassembly is predicted using Monte Carlo simulations. In the simulations, the specific effects of the chosen joining method, besides the actual joining of the parts of course, is often neglected. Some work has although been done on spot welding and spot welding sequences, see for example [1].

Welding simulation tools are used to calculate the thermal field, the evolution of microstructure and the stress-strain evolution during welding. Distortions and residual stresses caused by welding are often of interest when choosing a proper welding procedure and designing the welded structure. The use of welding simulations can reduce the amount of physical testing needed in the development of new welded products. 
Generally, it is important to strive for the best possible accuracy and precision in all kind of simulations, and of course, this is also of great importance in both variation simulations and welding simulations. Therefore, this work aims to combine variation simulation and welding simulation, by taking the effect of welding into consideration in the variation simulations and by applying the welding simulations to non-nominal parts. In that way, the quality of the simulations can be further improved, which leads to reduced risks of misjudgements and thereby also a reduced scrap rate. A reduced scrap rate will benefit sustainability, with respect to both economical and ecological aspects. Also the social sustainability is gained by increased use of virtual tools, since this usually implies improved working conditions.

Not much work has been done in the area of combining variation simulations and welding simulations. However, Lee et al. [2] have been studying weld distortion in tolerance simulations. They established, by using Finite Element Analysis (FEA), a database containing weld distortion data corresponding to a number of different welding parameters. This weld distortion was then retrieved from the database and used as a tolerance in the variation simulations. The welding distortion in the database was though based on simulations on nominal parts.

Xiong et al. [3] built a mathematical model for variation prediction taking locator errors, part errors and welding errors into account. The welding errors must however be specified as an input to the model, and the dependency between part error and the resulting welding error is consequently not taken into account.

\subsection{Variation simulations}

Variation simulation, together with other kind of analyses, such as stability analysis for evaluation of positioning systems and contribution analysis for distributing tolerances [4], form the basis of the evaluation and verification of a chosen concept. This is done in early stages of the product development cycle, often even before the first prototype is built.

Variation simulations are in many cases based on Direct Monte Carlo (DMC) simulation [5], where the statistical distributions for the parameters affecting a critical measure are defined. Given this input, a resulting value in a critical dimension can be computed. By repeating these steps a number of times, a distribution of the critical dimension can be approximated. The results are then compared to the predetermined requirements. The analysis utilizes a virtual assembly model with all mating conditions (locating schemes) defined. The method captures non-linearity's and allows any kind of distributions of input parameter variation.

Comparisons of different methods for variation simulations were done by Shen et. al [6]. Robustness evaluations and coupling analysis were reported in [7].

By incorporating FEA techniques also non-rigid tolerance analysis may be performed [8]. This analysis may be used to simulate how parts or subassemblies of non-rigid parts, such as sheet metal and plastic parts, behave after assembly. In non-rigid analysis, over-constrained locating schemes with more than six locators may be used and parts are allowed to bend and flex during assembly.
In this work, the software "Robust Design and Tolerancing" (RD\&T) [9] is used for the variation simulations. The software uses Monte Carlo simulations. An overview of functionalities provided in RD\&T is given by [4].

\subsection{Welding simulations}

The field of welding simulations/computational welding mechanics (CWM) has been well described by, for example, Goldak [10] and Lindgren [11-14]. The first attempts to compute distortions and residual stresses in simple welded 3D structures using CWM started in the beginning of the 1980s. Since then, several reports exist that show how welding distortions and residual stresses can be predicted using CWM [11-13]. Pahkamaa et. al [15] and Karlsson et. al [16] demonstrated how welding simulations can be used in a simulation driven design process. VrWeld [17], a CWM software developed by Goldak Technologies Inc. is used to conduct the welding simulations in this paper. Given CAD files of the parts being welded, a description of the welding procedure and material properties for the welded materials, VrWeld enables a designer to simulate the transient 3D temperature field, the evolution of microstructure, transient $3 \mathrm{D}$ displacement, stress and strain in the structure as it is being welded [17]. VrWeld solves the nonlinear, coupled 3D transient partial differential equations (PDEs) for heat flow (conservation of energy), microstructure evolution and stress-strain evolution (conservation of momentum) [18]. The results can then be post processed and visualized in VrWeld.

\subsection{Scope of the paper}

In this paper, variation simulations and welding simulations are combined, i.e. welding simulations are applied on a population of non-nominal parts.

An overview of both variation simulations and welding simulations was given in the introduction. How the both kind of simulations can be combined are explained in Section 2. The suggested method is applied to a case study and the results from this case study are described in Section 3. The conclusions are found in Section 4.

\section{COMBINING VARIATION SIMULATION AND WELDING SIMULATIONS}

This chapter presents the methodology which is used in this paper to combine variation simulations with welding simulations. The parts being welded are modeled in a CAD software, in this case Siemens NX 7. The parts are then exported to RD\&T in VRML2 format (Virtual Reality Modelling Language) and to VrWeld in STL format (StereoLithography).

In RD\&T a virtual assembly model is created. This model describes what the parts look like and how they are positioned in the assembly fixture. Thereafter, tolerances are added to the locating points in the fixtures. Those tolerances describe variation in the contact between the locator in the fixture and the corresponding contact area of the part. These variations can origin from the fixture as well as from the part itself. Those sources of variation are usually split into different tolerances, but in this initial research those are combined in one tolerance. In future work, separate tolerances for fixture and part variation and also variation in contact areas between parts may be considered. 
When the assembly model is prepared, a number of Monte Carlo iterations are executed. The result of each iteration can be described by a rigid body transformation (RBT), consisting of one translation and one rotation. Each point $\boldsymbol{a}$ $=\left(\begin{array}{lll}a_{x} & a_{y} & a_{z}\end{array}\right)^{\mathrm{T}}$ in the original coordinate system can be transformed to the new, disturbed position $\boldsymbol{a}^{\boldsymbol{\prime}}$ by applying the translation and rotation matrices:

$$
\boldsymbol{a}^{\prime}=\boldsymbol{R a}+\boldsymbol{t}
$$

For a rigid body transformation, the rotation matrix $\boldsymbol{R}$ and the translation vector $\boldsymbol{t}$ can be uniquely determined [19].

This information, describing non-nominal parts as rigid body transformations of the nominal parts, are exported to VrWeld in a comma-separated value text document (CSV).

In VrWeld, a base project is created. The base project is created by importing the STL-files, defining welding procedures and material models, defining weld paths and weld joints, meshing parts and weld joints, setting up constraints and boundary conditions. A copy of this base project is then created for each of the cases generated in RD\&T. The rigid body transformations generated in RD\&T are applied on the meshes for each part in the project. Since the weld joint is connected to at least two parts, it will be deformed as the two parts moves with respect to each other. This deformation is calculated by letting the weld joint nodes connected to the moved parts be moved, and then solving the linear deformation that it results in. All stresses and strains are then set to be zero at this new position. If the movements of the parts are too big it might deform the weld joint so much that the weld joint elements becomes badly shaped, or it might give the weld joint an unwanted shape. In this case, the weld joint has to be generated after the RBT is performed. Once the simulation models for each case is prepared (nominal case included), they are solved in parallel using multicore processors. The process of preparing each copy of the base project can be automated by using the Multiproject function in VrWeld. Given the RBT-matrix for each part, this function will automatically create a copy for each of the predetermined cases and update the meshes. Then it's just a matter of starting the simulations. The results are post processed and exported back to RD\&T for further analysis. The results are exported in three meshes, nominal mesh, disturbed mesh and deformed mesh after welding. The resulting deformation is exported from VrWeld to RD\&T as an VTK mesh. In RD\&T the data is gathered and analyzed. For each node the standard deviation and mean value for all iterations are calculated. The results are illustrated using color coding. Figure 1 shows how the data is transported between the softwares.

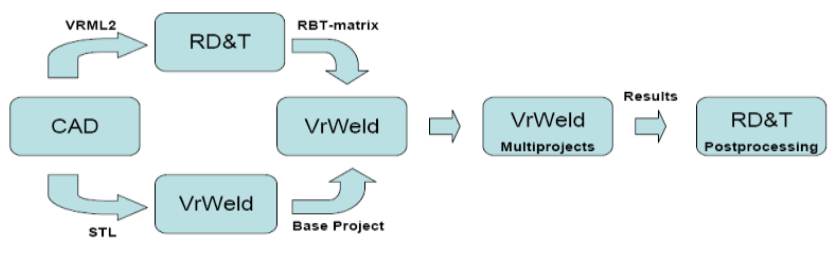

Figure 1. Flow chart.

\section{CASE STUDY}

A simple T-joint case is simulated to demonstrate the flow of data when combining variation simulations with welding simulations. Deo and Michaleris [20] conducted experiments and welding simulations on " $T$ " shaped stiffeners commonly used in the shipbuilding industry. The experimental setup referred to as "Case 2" in [20] will be used for this paper. The case consists of two A36 steel plates joined by a $\mathrm{T}$-joint configuration, i.e. a fillet weld on each side of the stiffener. The plates are tack welded at the start and stop end of the stiffener prior to welding.

In RD\&T tolerances are applied to the locators of both parts. In each locator, a tolerance where $8 \sigma=0.7$ is applied. This tolerance can be regarded as a common tolerance, containing both variation in the fixture due to wear or lack of repeatability and variation in areas meeting the locator on the parts. The tolerances are centered around nominal values. A sample of 37 details, using those non-nominal conditions that the tolerances imply, were created using Monte Carlo simulations. Of course, it would be desirable with a larger sample size, but due to computational expensive welding simulations, the sample size was limited to 37 in this first experiment.

The variations generated in RD\&T are applied prior to tack welding to simulate variations in the tack welding fixture.

The plates are 48" (1219.2 mm) long, 4" (101.6 mm) wide and $3 / 16^{\prime \prime}(4.8 \mathrm{~mm})$ thick. The weld size is $5 / 32 "(4.0 \mathrm{~mm})$. The weld travelling speed is $6.35 \mathrm{~mm} / \mathrm{s}$ and the welding power is $4800 \mathrm{~W}$ ( $24 \mathrm{~V}$ and $200 \mathrm{~A})$. The welding setup consists of a welding gun on each side of the stiffener. The guns are 3.5 " $(88.9 \mathrm{~mm})$ offset from each other with one gun following the other. Additional information about the experimental setup can be found in [20]. The base project simulation setup can be seen in Figure 2. The simulation mesh consists of 20478 eight-node brick and six-node prism elements. Figure 2 also shows the simulation constraints, this approach prevents rigid body motions by locking 6 degrees of freedoms by constraining three nodes.

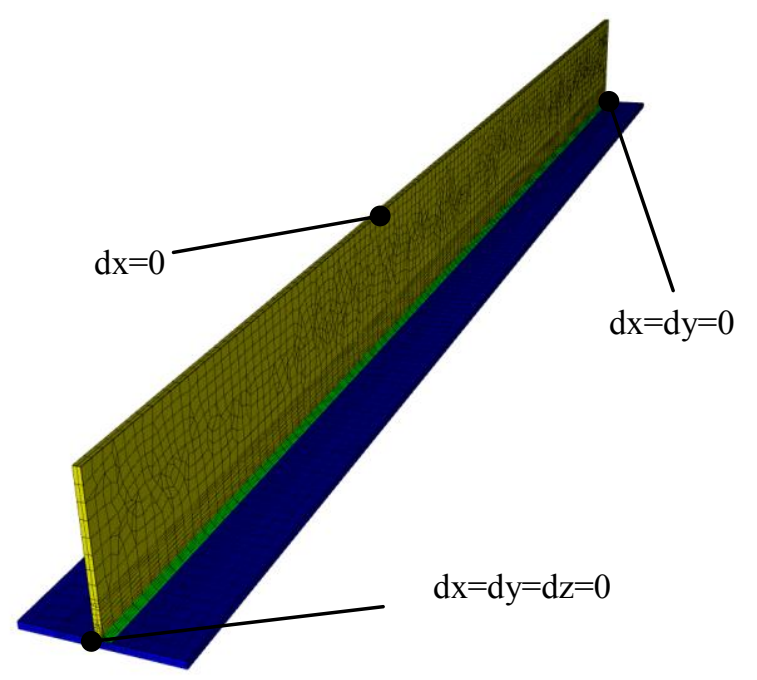

Figure 2. Simulation mesh and constraints.

The heat input is simulated using a double ellipsoid [10] moving heat source, see Figure 3. The heat input parameters $\left(a_{1}, a_{2}, b\right.$ and $\left.c\right)$ describes the shape of the heat input. The heat input parameters used for both welding guns can be found in Table 1. The A36 steel material model presented by Deo et. al [21] has been implemented in VrWeld and is used in the simulations. 


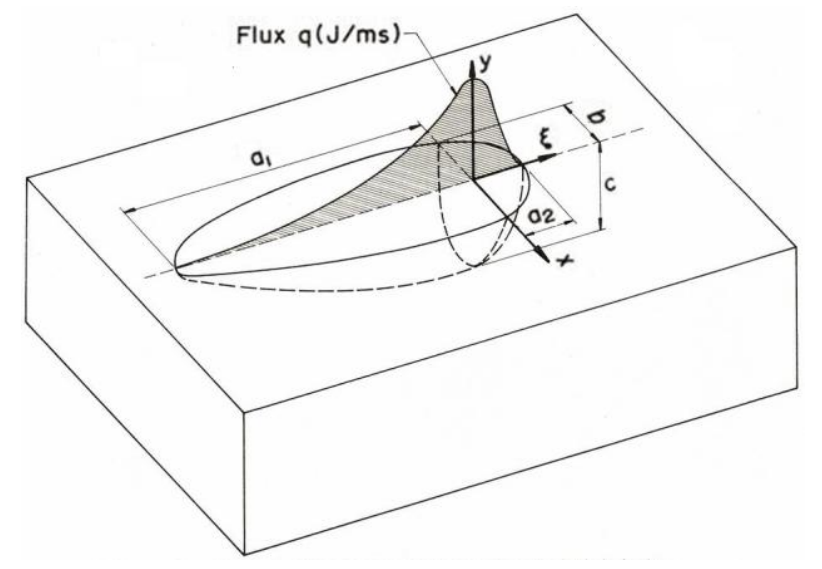

Figure 3. Double ellipsoid heat source with Gaussian heat distribution. [10]

Table 1. Heat input parameters.

\begin{tabular}{ll}
\hline Parameter & Value \\
\hline Heat input model & $\mathrm{a}_{1}=12 \mathrm{~mm}$ \\
(Double Ellipsoid Moving & $\mathrm{a}_{2}=6 \mathrm{~mm}$ \\
Heat Source) & $\mathrm{b}=2.5 \mathrm{~mm}$ \\
& $\mathrm{c}=2.5 \mathrm{~mm}$ \\
\hline Welding Net Power & $4800 \mathrm{~W}(24 \mathrm{~V}, 200 \mathrm{~A})$ \\
\hline Welding Efficiency & $85 \%$ \\
\hline Welding Speed & $6.35 \mathrm{~mm} / \mathrm{s}$ \\
\hline
\end{tabular}

Figure 4 shows how the weld joint has deformed because of the new positions of the welded parts. The shape of the weld joint and its elements were considered good enough and didn't need to be regenerated or remeshed.

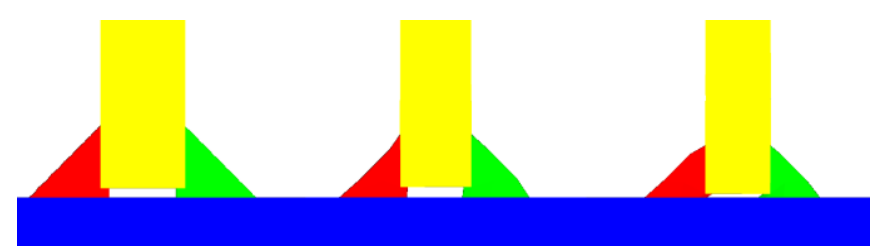

Figure 4. Weld joint before (left) and after RBT, two cases (centre and right).

Figure 5 shows the resulting deformation after welding of the nominal case. The simulation results show good agreement with deformation and residual stress measurements conducted by Deo and Michaleris [20]. The bowing at the center of the beam is predicted to be $4.7 \mathrm{~mm}$, compared to $5.7 \mathrm{~mm}$ obtained in the experiment. Figure 6 shows a comparison between predicted and measured residual stress, though more residual stress measurements would be needed to make a sufficient comparison.

As mentioned earlier, a sample containing 37 different nonnominal conditions for the parts was created using Monte Carlo simulation. For each set of non-nominal condition, welding simulation is applied. The goal is to investigate if there is a difference, larger than the applied tolerance, between welding simulations applied to nominal conditions and welding simulations applied to non-nominal conditions. It is also of interest to investigate how the influence of welding affects the variation simulations.

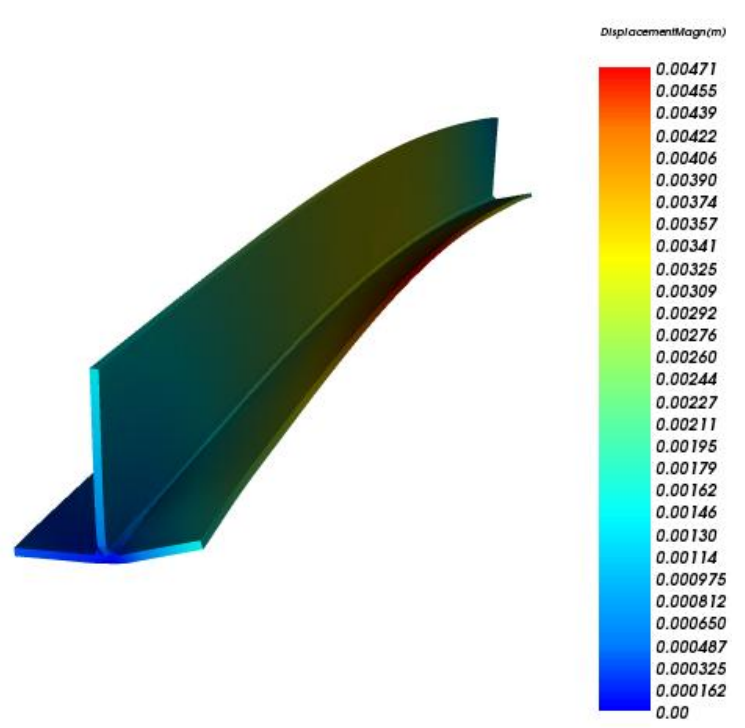

Figure 5. Resulting deformation of nominal case. Deformation enhanced 10X.

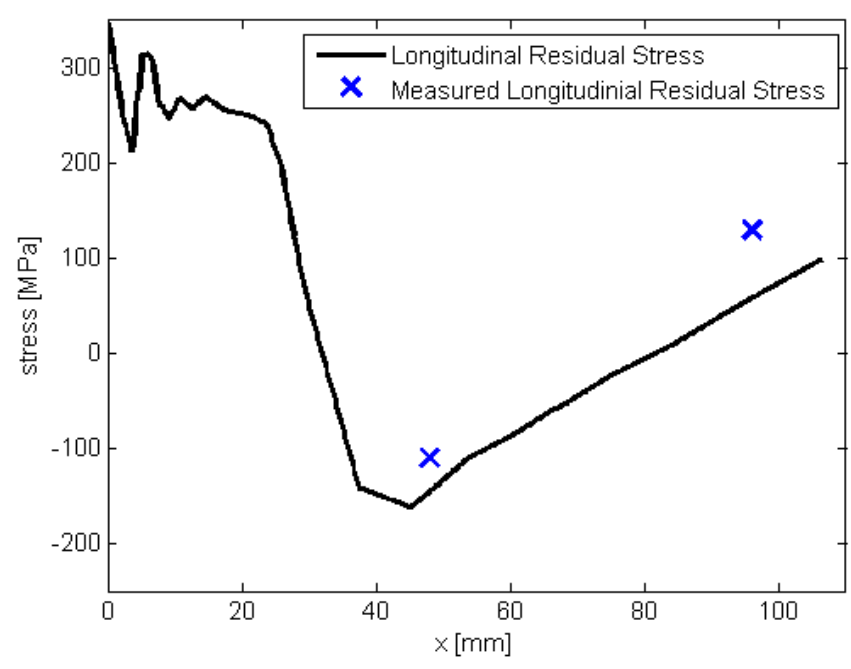

Figure 6. Comparison between predicted residual stress and experimental values.

In Figure 7 the results concerning deviation or mean value can be seen. In Figure 7A, the deviation from nominal after welding using nominal conditions can be seen. In Figure 7B the mean value of the deviations from nominal for nonnominal conditions before welding is shown. Since the tolerances are centred on zero, the mean deviation is very small; observe the different scale in this picture compared to Figure 7A and Figure 7C. In Figure 7C the mean value of the deviations for non-nominal conditions after welding is illustrated. Since the mean effect of the tolerances are almost negligible, Figure 7A and Figure 7C are very similar. 

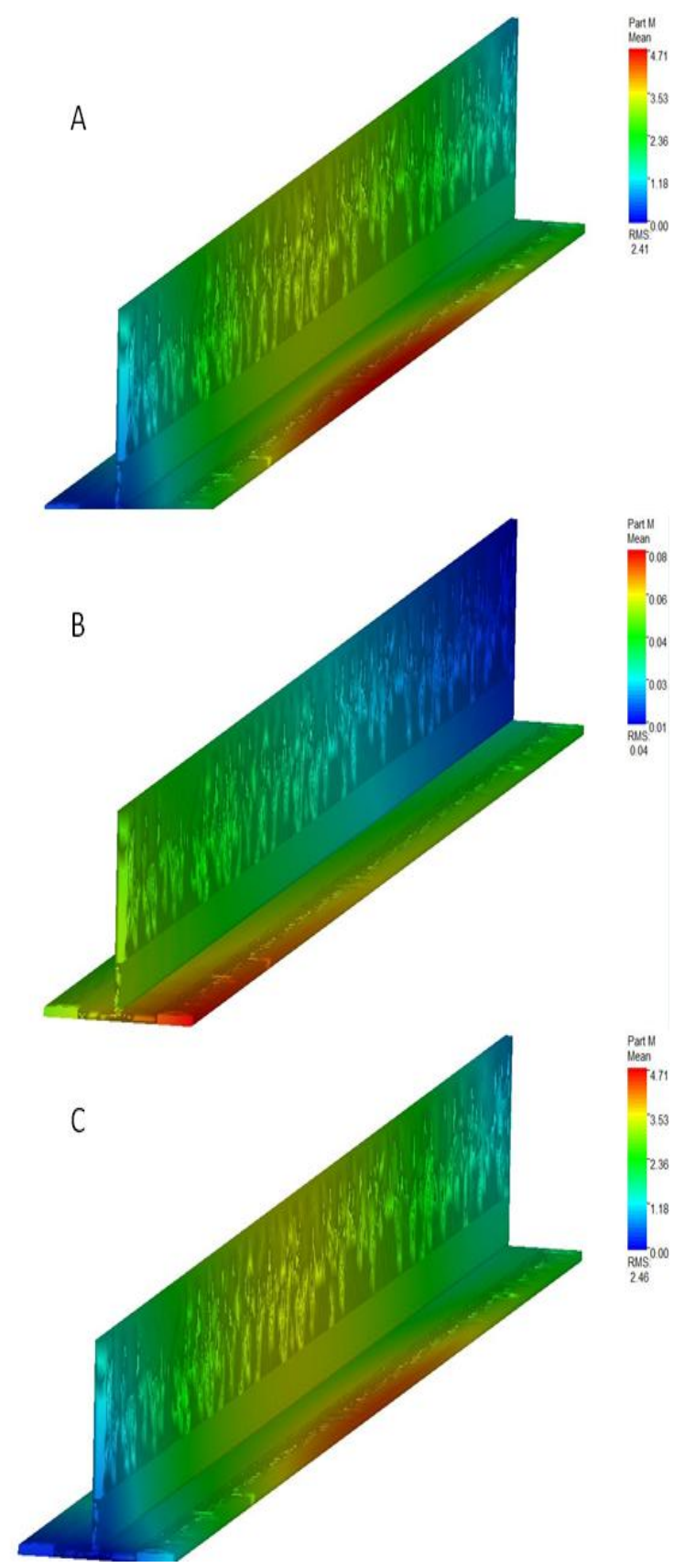

Figure 7. A) The effect of welding under nominal condition. B) The mean value for non-nominal conditions before welding. C) The mean value for nonnominal conditions after welding.

To study the effect of welding for non-nominal conditions compared to welding for nominal conditions more closely, the behaviour in five different nodes for one of the 37 set of non-nominal conditions is investigated. Only one iteration is considered and therefore it is only possible to investigate the mean value deviation, not any variation. The positions of the nodes considered are shown in Figure 8.

In Figure 9, the deviation in $\mathrm{x}-$, $\mathrm{y}$ - and $\mathrm{z}$-direction in each node after welding using non-nominal conditions are shown (red bar) as well as the deviation after welding using nominal conditions (green bar). Further, the sizes of the added deviations (the blue bar) in each node are illustrated.

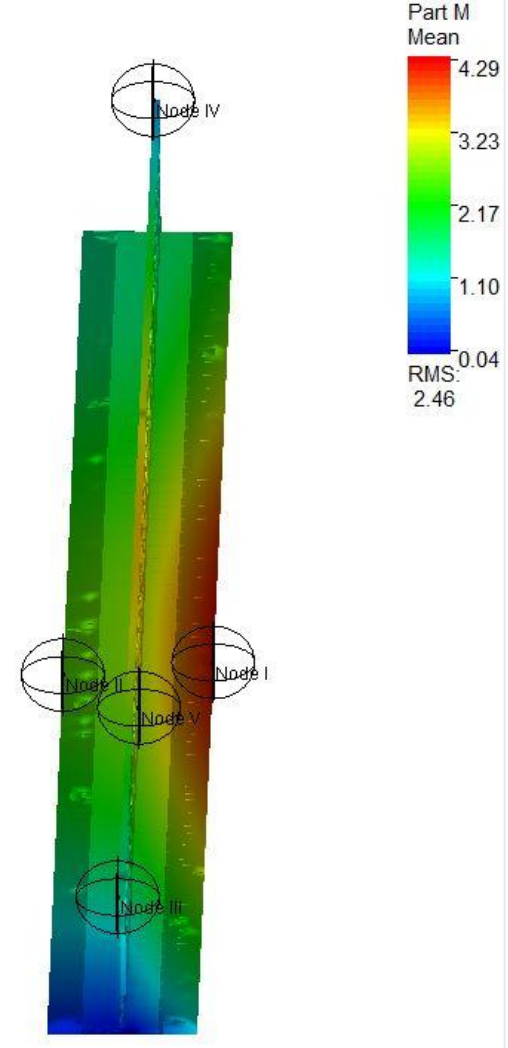

Figure 8. The position of the nodes investigated in Figure 9

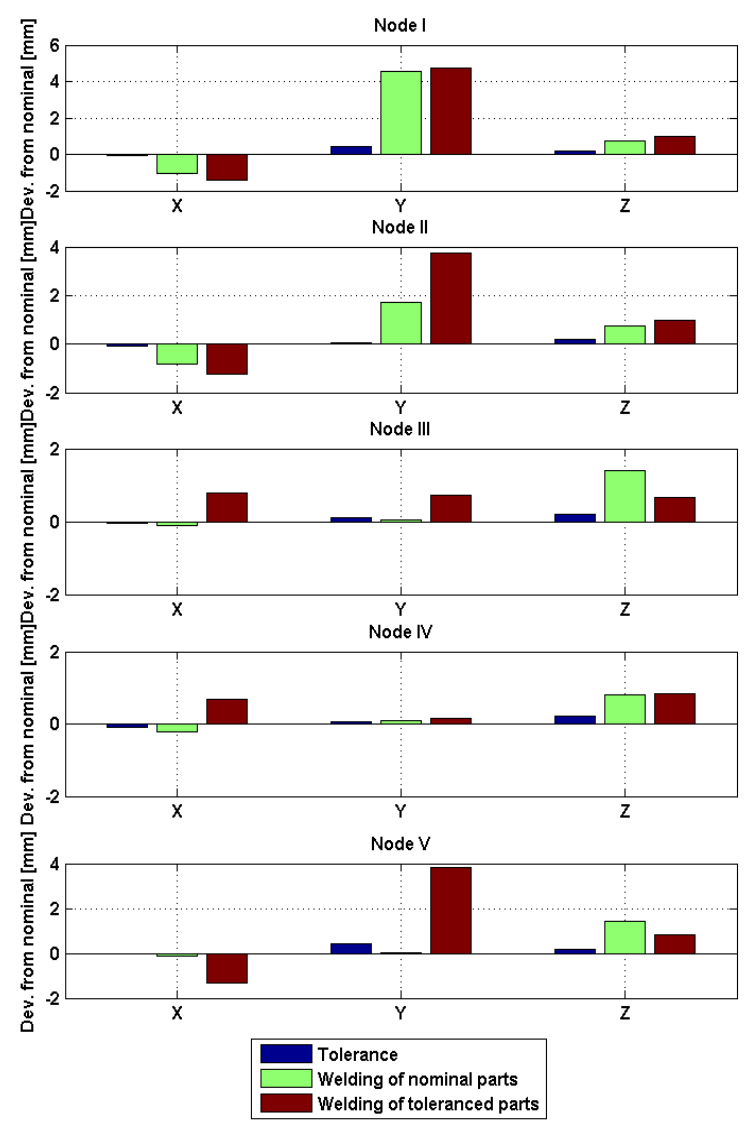

Figure 9. The final results compared to welding of nominal parts and to the tolerance applied. 
The figure shows that the difference between welding using non-nominal conditions and nominal conditions can be considerable. For example, in Node $\mathrm{V}$ in $\mathrm{Y}$-direction, the welding for nominal conditions results in almost no deviation from nominal at all, while the deviation for nonnominal condition amount to almost $4 \mathrm{~mm}$. For some other nodes and directions, there are only minor differences. It seems to be difficult to find some pattern in the behaviours, and of course, the behaviour in one node depends on what happens in other nodes of the assembly.

To summarize, there are large differences between the effect of welding applied to nominal conditions and the effect of welding applied to non-nominal conditions and it is important to be aware of these differences when conducting welding simulations.

In Figure 10 the values of $6 s$ are illustrated using color coding. In this figure, it is not possible to show the variation of welding for nominal conditions, since there is only one nominal assembly, and consequently no variation between different assemblies. All welding conditions besides the tolerances are constant during this study. It can though be seen that the amount of variation increases from Figure 10A, where $6 s$ for all non-nominal conditions before welding is shown, to Figure 10B, where $6 s$ for non-nominal conditions after welding is illustrated. It is accordingly possible to establish that the welding procedure increase the amount of variation and also that the added variation is not distributed evenly all over the assembly. For example, the upper left corners in Figure 10A and Figure 10B are almost the same, while the variation has increased significantly in the area that is red in Figure 10B.

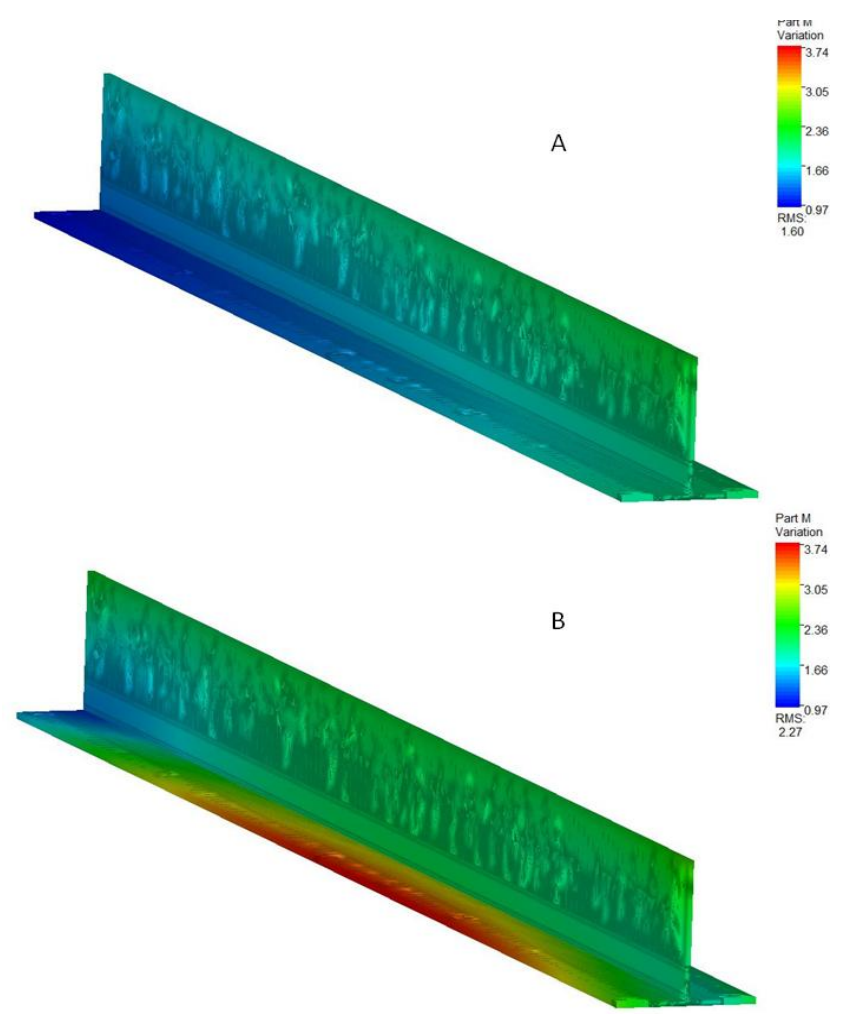

Figure 10. A) $6 s$ for non-nominal conditions before welding. B) $6 s$ for non-nominal conditions after welding.
Figure 10 does show the importance of including the effect of welding in variation simulations, since it leads to a significant increase of the level of variation in the final assembly.

\section{CONCLUSIONS}

The geometrical variations on parts and the influence of heating and cooling due to the welding process both strongly affect the final geometrical outcome of an assembly. Those two aspects are however usually simulated separately, which may give misleading results. In this work, variation simulation and welding simulation are combined.

The method is applied to a case study and the results are analyzed with respect to both variation and deviations from nominal values.

For the deviation, there are evident differences in deviation from nominal when the welding simulations are applied to parts under nominal conditions compared to when they are applied to parts under non-nominal conditions (due to tolerances added in the variation simulation). This motivate why it is not enough to carry out welding simulations on nominal parts.

When comparing the levels of variation before and after welding, it is also clear that it is important to include the effect of welding in the variation simulations to achieve a realistic result.

\section{ACKNOWLEDGEMENT}

This work was financed by VINNOVA (the Swedish Governmental Agency for Innovation Systems) and carried out in parallel with the work in the (also VINNOVA funded) Faste Laboratory and Wingquist Laboratory. The VrWeld software used for the simulations in this research was provided by Goldak Technologies Inc. The RD\&T software used for the simulations in this research was provided by RD\&T Technology.

\section{REFERENCES}

[1] Wärmefjord, K., R. Söderberg and L. Lindkvist, Strategies for Optimization of Spot Welding Sequence With Respect to Geometrical Variation in Sheet Metal Assemblies. Submmitted to Journal of Manufacturing Science and Engineering, 2010.

[2] Lee, D., K.E. Kwon, J. Lee, H. Jee, H. Yim, S.W. Cho, J.-G. Shin and G. Lee, Tolerance Analysis Considering Weld Distortion by Use of Pregenerated Database. Journal of Manufacturing Science and Engineering, 2009. 131(4): p. 041012-8.

[3] Xiong, C., Y. Rong, R.P. Koganti, M.J. Zaluzec and N. Wang, Geometric variation prediction in automotive assembling. Assembly Automation, 2002. 22(3): p. 260-269.

[4] Söderberg, R., L. Lindkvist and J. Carlsson, Virtual Geometry Assurance for Effective Product Realization, in 1st Nordic Conference on Product Lifecycle Management - NordPLM'06. 2006: Göteborg.

[5] Gao, J., K.W. Chase and S.P. Magleby, Comparision of Assembly Tolerance Analysis by the Direct Linearization and Modified Monte Carlo Simulation 
Methods, in ASME Design Engineering Technical Conference. 2009: Boston, USA. p. 353-360.

[6] Shen, Z., G. Ameta, J.J. Shah and J.K. Davidson, $A$ Comparative Study Of Tolerance Analysis Methods. Journal of Computing and Information Science in Engineering, 2005. 5(3): p. 247-256.

[7] Söderberg, R. and L. Lindkvist, Computer Aided Assembly Robustness Evaluation. Journal of Engineering Design, 1999. 10(2): p. 165-181.

[8] Wärmefjord, K., R. Söderberg and L. Lindkvist, Tolerance simulation of compliant sheet metal assemblies using automatic node-based contact detection, in Proc. of IMECE200. 2008: Boston, USA.

[9] RD\&T Manual. 2009.

[10] Goldak, J. and M. Akhlagi, Computational welding mechanics. 2005: Springer.

[11] Lindgren, L.E., Finite element modeling and simulation of welding. Part 1: Increased complexity. Journal of Thermal Stresses, 2001. 24(2): p. 141-192.

[12] Lindgren, L.-E., Finite element modeling and simulation of welding. Part 2: Improved material modeling. Journal of Thermal Stresses, 2001. 24(3): p. $195-231$.

[13] Lindgren, L.E., Finite element modeling and simulation of welding. Part 3: Efficiency and integration. Journal of Thermal Stresses, 2001. 24(4): p. 305-334.

[14] Lindgren, L.-E., Computational welding mechanics : thermomechanical and microstructructural simulations. 2007, Boca Raton, Fla. :: CRC Press ;.

[15] Pahkamaa, A., L. Karlsson, J. Pavasson, M. Karlberg, M. Näsström and J. Goldak, A Method to Improve Efficiency in Welding Simulations for Simulation Driven Design, in ASME 2010 International Design Engineering Technical Conference \& Computers and Information in Engineering Conference. 2010: Montreal, Quebec, Canada.

[16] Karlsson, L., A. Pahkamaa, M. Karlberg, M. Löfstrand, J. Goldak and J. Pavasson, Mechanics of Materials and Structures - a Simulation-Driven Design Approach. Accepted for publication in Journal of Mechanics of Materials and Structures.

[17] http://www.goldaktec.com/. 2010; Available from: http://www.goldaktec.com/.

[18] Goldak, J., Distortion and Residual Stress in Welds: The Next Generation, in 8th International Conference on Trends in Welding Research. 2008: Pine Mountain, Georgia.

[19] Corben, H. C. and P. Stehle, Classical Mechanics, 1994: Dover Publications, New York

[20] Deo, M.V. and P. Michaleris, Experimental Verification of Distortion Analysis of Welded Stiffeners. Journal of Ship Production, 2002. 18(4): p. 216-225.

[21] Deo, M.V., P. Michaleris and J. Sun, Prediction of buckling distortion of welded structures Science and Technology of Welding \& Joining, 2003. 8(1): p. 5561. 